Article

\title{
Integrated Energy Micro-Grid Planning Using Electricity, Heating and Cooling Demands
}

\author{
He Huang ${ }^{1, *}$, DaPeng Liang ${ }^{1}$ and Zhen Tong ${ }^{2}$ \\ 1 School of Management, Harbin Institute of Technology, Harbin 150001, China; ldp0920@hit.edu.cn \\ 2 Electric Power Development Research Institute, China Electricity Council, Beijing 100761, China; \\ tongzhen@cec.org.cn \\ * Correspondence: superrio@yeah.net; Tel.: +86-138-1041-3900
}

Received: 12 September 2018; Accepted: 17 October 2018; Published: 18 October 2018

\begin{abstract}
Many research works have demonstrated that taking the combined cooling, heating and power system (CCHP) as the core equipment, an integrated energy system (IES), which provides multiple energy flows by a combination of different energy production equipment can bring obvious benefit to energy efficiency, $\mathrm{CO}_{2}$ emission reduction and operational economy in urban areas. Compared with isolated IES, an integrated energy micro-grid (IEMG) which is formed by connecting multiple regions' IES together, through a distribution and thermal network, can further improve the reliability, flexibility, cleanliness and the economy of a regional energy supply. Based on the existing IES model, this paper describes the basic structure of IEMG and built an IEMG planning model. The planning was based on the mixed integer linear programming. Economically, construction planning configuration are calculated by using known electricity, heating and cooling loads information and the given multiple equipment selection schemes. Finally, the model is validated by a case study, which includes heating, cooling, transitional and extreme load scenarios, proved the feasibility of planning model. The results show that the application of IEMG can effectively improve the economy of a regional energy supply.
\end{abstract}

Keywords: energy internet; multi-energy complementary; integrated energy systems; distribution network planning

\section{Introduction}

Increasing pressure on energy resources endowment and environmental problems resulting from the use of the energy internet (EI) are a major focus of energy researchers and practitioners [1]. Combined cooling, heating, and power unit (CCHP) technology integrates production of power from electrical and thermal systems, and solves problems caused by their separate decision-making frameworks. With support of a CCHP, an integrated energy systems (IES) can provide multiple energy flows (electricity, heating, steam, cooling, and desalination) by combining different energy production equipment (natural gas, solar, wind, etc.). It has become widely accepted as one of the most efficient examples of integration of multiple energy sources [2]. The biggest benefit of IES is that different kinds of energy production systems are no longer planned separately or operated independently. It thus takes the overall process of energy production-from generation and transmission to consumption-into full consideration during the stages of planning, construction, and operation [3,4]. Numerous cases testify to the strength of IES (with a CCHP at its core) in urban areas where it improves primary energy use efficiency, $\mathrm{CO}_{2}$ emission reduction, and the operational economy $[5,6]$. Its position in the energy network has become increasingly important.

IES theories can be summarized as electricity-heat IES and electricity-gas-heat IES [7]. The author of [8] expounded the importance of electricity and heating networks combination analysis. The physical 
interaction between electricity and heating networks are discussed by Pan et al. in [9]. Zeng et al. [10] designed an analysis framework considering bi-directional energy conversion, which unified the powerand gas-flow of an electricity-gas IES. The author of [11] explored the impacts of gas composition on both electricity and gas networks by a decomposed method of power- and gas-flow analysis. References [12,13] proposed a steady-state energy-flow analysis of IES containing electricity, gas and heat based on the Newton-Raphson method. Some other studies have focused on expanding the components of IES. An IES model based on a micro-grid-including the combination of the CCHP and renewable energies - was established [14,15], and the benefits of the model for increasing the utilization rate of renewable energy and reducing the energy consumption of the CCHP were proven. The authors in [16] describes an IES operation optimization model including photovoltaic and battery energy storage, and illustrated that battery lifetime loss is an inevitable factor in the optimization model.

On the basis of previous studies of IES analysis and modelling, planning is also one of the steady-state research directions. An electricity distribution lines and elements expansion model, which provides optimal reconfiguration in electricity and natural gas distribution systems within energy hubs, is proposed in [17]. Multi-area and multistage gas and electricity infrastructures integrate the expansion planning model from the central decision maker perspective and is formulated in [18]. Based on the planned power generating units, Ref. [19] built a centralized expansion planning model that integrates gas and electricity distribution networks. A joint expansion planning model of combined gas and electricity networks with the objective function of maximizing the social welfare is presented in [20]. A multi-period integrated framework incorporates a three-level procedure to solve the generation, transmission and natural gas grid expansion planning for large-scale systems is introduced in [21]. In the content of uncertainties from increasing utilization of natural gas in an electric power system, a novel expansion co-planning framework is proposed in [22] to address the integrated gas-electricity expansion planning that considers maximization of the cost/benefit ratio with a market price of gas and electricity as several scenarios. An integrated electricity and natural gas transportation system planning algorithm that is based on a two-stage robust optimization problem is provided in [23] for enhancing the power grid resilience in extreme conditions. In this model, a variable uncertainty set is involved to describe the interactions among power grid expansion states and extreme events and its case study result shows the benefit of integrated planning on improving power grid resilience. The centralized expansion planning model upon a two-stage stochastic optimization framework is established in [24], and the model provides the tradeoff of building natural gas facilities versus electric facilities under the uncertainty of demand growth. A long-term planning model of gas distribution pipelines, gas-fired power generators, and capacitor banks is presented in [25], which is solved by a sequential planning approach, and its result proves a relationship between the expansion plans and the reliability policies of a distribution utility. A co-expansion planning of gas and electricity systems based on a multi-attribute decision-making method (MADM) is introduced in [26] by the analytical hierarchy process of the central entity and privacy of gas and electricity energy parties, and the model demonstrated the effectiveness of the proposed MADM method.

Previous research on the planning and operation optimization of IES have laid a foundation. Thus, based on the theory of electricity-heat IES, this paper proposes an integrated energy micro-grid model containing distributed energy resources, and taking into account various load conditions. It can be used in IEMG planning and operation optimization. To prove the validity of the model, an IEMG planning framework by using electricity, heating and cooling demands are introduced. Through a mixed integer linear programming method (MILP), the economy of construction and operation of IEMG is optimized and analyzed. The main characteristics are as follows. Firstly, from the perspective of regional integrated energy suppliers, comprehensive planning was carried out to fulfill the power electricity, heating, and cooling loads in multiple regions, including economic analysis of the construction and operation costs of IEMG. Secondly, more equipment options are available for the planning selection, including the addition of solar power, substation expansion, and additional CCHPs, gas boilers, an absorption chiller, or air conditioning. Thirdly, in terms of the operational 
strategy, the model divides the energy cycle into winter, summer, and an interim period, according to changes in load demands. An extreme load scenario was added to further guarantee the accuracy and reliability of the planning results. Finally, this paper verifies the IEMG planning model with practical examples to prove its significance in guiding the construction and operation of IEMG for regional integrated energy suppliers.

\section{The Integrated Energy Micro-Grid}

\subsection{Structure of the Integrated Energy System (IES) and the Integrated Energy Micro-Grid (IEMG)}

IES usually consists of a CCHP, distributed power sources (adjustable and/or non-adjustable), the electrical load, heating load, cooling load, thermal network, and the electrical network. It can also be connected to the external power grid by a transformer substation. The Busbar structure for IES is shown in Figure 1 [27].

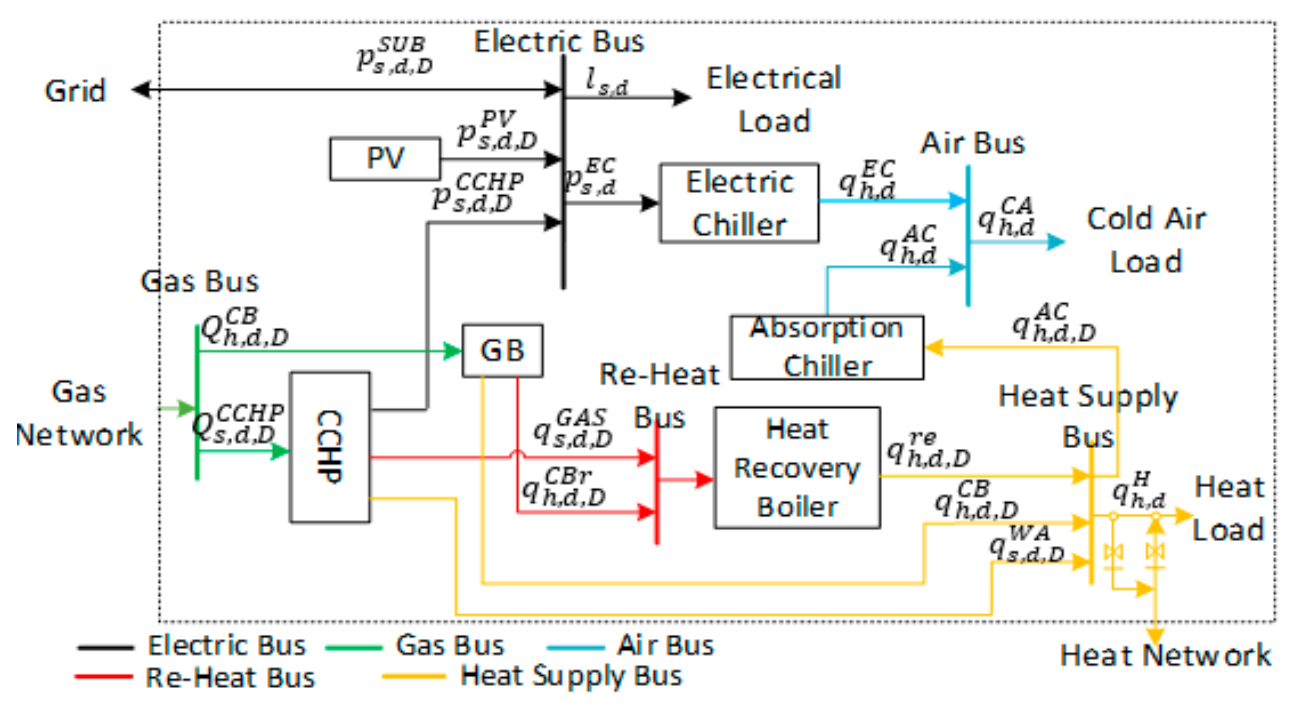

Figure 1. The Busbar structure for the integrated energy systems (IES).

In IES, the demands of electricity, heating, and cooling loads can be satisfied simultaneously. The electricity demand is generated by the CCHP, distributed power resources (photovoltaic (PV) cells, for example), and an external grid (when the load demand exceeds the total electricity capacity), and so on. The heating demands are fulfilled by the CCHP and the gas boiler, and the cooling demands are met by both an electric chiller and an absorption chiller. The heat recovery boiler (HRB) acts as a waste heat recovery facility, and can collect the waste heat generated by both the CCHP and the gas boiler, which can significantly improve the amount of heat used in the system. The power grid, the thermal equipment linking to the link energy production equipment, and the different loads operate together to achieve energy circulation through the whole system. It is necessary to emphasize that the interaction between the CCHP and the thermal network is bidirectional, so there is a switch apparatus between them to achieve directional selectivity. The heat networks of different regions can also transfer heat through a switch apparatus between the heat exchanger and the heat load.

A schematic diagram of the IEMG is shown in Figure 2. The IEGM connects several regions' IES (here called the subarea of the IEMG) by a micro-grid, a heat network, and a natural gas pipeline network, to make a scheduling balance within the whole region possible. Thus, the IEMG regards the multiple IES as a controllable whole-they can be safely connected to the low-voltage distribution grid and operate in a flexible manner. Meanwhile, through the coordinated control of the equipment in these regions, the IEMG can provide a more economical, efficient, and reliable supply of energy for different kinds of loads. Furthermore, it can connect to the external power grid and the thermal network, and can thus purchase electricity and heat energy when the overall output in the region 
is insufficient, or sell surplus energy to external buyers. It can, therefore, significantly improve the economic efficiency for integrated energy suppliers. To summarize, due to the multi-region and multi-energy complementation, the electricity reliability, economical efficiency, and comprehensive utilization rate of energy within the region of the IEMG is effectively improved.

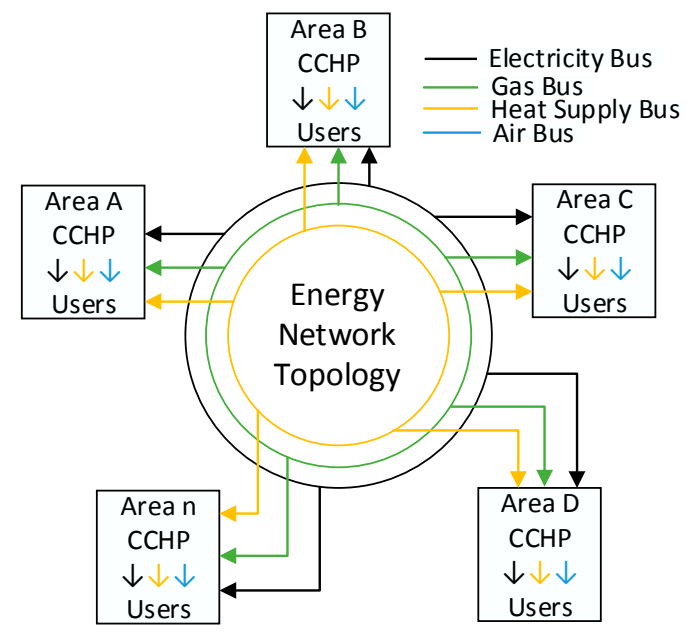

Figure 2. Diagram of the integrated energy micro-grid (IEMG).

\subsection{The Principle Elements of the IEMG}

The IEMG model in this paper includes three main parts: energy generation, the transformation network, and loads. Generation refers to the electricity, heat, and other energy supply equipment, including the gas combustion engine, CCHP, photovoltaics, substation, and boiler. The network denotes the electrical power grid and the thermal network. The loads are the electrical, heating, and cooling loads. This section models these devices mathematically.

\subsubsection{The Generation Equipment}

(1) Combined Cooling, Heating and Power System (CCHP)

In this paper, the CCHP is built using a constant efficiency model, and the relationship between its thermoelectric power and fuel consumption is established through approximation of a linear function $[28,29]$.

The fuel combustion of the CCHP can be calculated by the following:

$$
Q_{s, d, D}^{C C H P}=V_{s, d, D}^{C C H P} \theta_{N G} / 3.6
$$

Accordingly, its electrical output is:

$$
p_{s, d, D}^{C C H P}=\alpha_{d, D}^{G E} Q_{s, d, D}^{C C H P}+\beta_{d, D}^{G E}
$$

The net calorific value of the waste heat is:

$$
q_{s, d, D}^{G A S}=\alpha_{d, D}^{G A S} Q_{s, d, D}^{C C H P}+\beta_{d, D}^{G A S}
$$

The net calorific value of the jacket-cooling water is:

$$
q_{s, d, D}^{W A}=\alpha_{d, D}^{W A} Q_{s, d, D}^{C C H P}+\beta_{d, D}^{W A}
$$

The expression of fuel combustion describes the total calorific value $Q_{s, d, D}^{C C H P}$ converted from the combustion inflow $V_{s, d, D}^{C C H P}$ (unit: $\mathrm{m}^{3} / \mathrm{h}$ ) per unit time, where $\theta_{N G}$ is the average calorific value (which is a constant, $32.967 \mathrm{MJ} / \mathrm{m}^{3}$ for natural gas). The other three expressions describe the electrical power, 
and the available calorific value in the waste heat generated by the CCHP. In these equations, $p_{s, d, D}^{C C H P}$ is the power output of the CCHP; $q_{s, d, D}^{G A S}$ and $q_{s, d, D}^{W A}$ are the net calorific values of the waste heat and the jacket-cooling water, respectively, in $\mathrm{kW}$. The parameters $\alpha$ and $\beta$ are two known coefficients that are used to fit the performance of the CCHP. In practice, the output of the CCHP is subject to other technical constraints, including the service life of the unit, the maximum and minimum output limitation, the ramp rate of the unit, the minimum continuous running time, and the minimum continuous downtime. These constraints and parameters are selected differently during the different optimizing purposes. The main purpose of this paper is the planning and optimization of the IEMG. Therefore, the specific constraints and parameter selection will be detailed later.

(2) Distributed Generation

The distributed generation in this paper is the PV power generation system, and the mathematical model applied is as follows. The output of a PV system is affected by weather, temperature, and solar illumination. If the PV output is $p_{s, d, D}^{P V}$, it can be modeled as:

$$
p_{s, d, D}^{P V}=\frac{\zeta_{t}}{\zeta_{t, s}} A \cdot f_{p v} \eta\left[1+\alpha_{p}\left(T_{s}-T_{s t c}\right)\right]
$$

where $\zeta_{t}$ is the actual illumination intensity during the $t$ th hour $(\mathrm{kW}) ; \zeta_{t, s}$ is the illumination intensity under standard conditions; $A$ is the total area of the PV panels, which is: $A=\sum_{m=1}^{M} A_{m}$, in which, $A_{m}$ is the area of a single panel; $f_{p v}$ is the power derating factor of the PV system, denoting the ratio of the actual output power to the rated output power, which is used to represent the power loss caused by dirt, rainwater, or snow on the PV panels, and by the aging of the panels (its value here is taken to be 0.9); $\eta$ is the overall conversion efficiency of the PV panels: $\eta=\frac{1}{A} \sum_{m=1}^{M} A_{m} \eta_{m}$, in which $\eta_{m}$ is the conversion efficiency of a single panel $(\mathrm{kW}) ; \alpha_{p}$ is the power temperature coefficient $\left(\% /{ }^{\circ} \mathrm{C}\right)$ (which is generally -0.47$) ; T_{s t c}$ is the reference temperature of the PV generation system measured under standard conditions $\left(25^{\circ} \mathrm{C}\right.$ here); $T_{s}$ can be calculated by:

$$
T_{s}=T_{a}+0.0138\left(1+0.031 T_{a}\right)\left(1-0.042 v_{p v}\right) \cdot A
$$

where $T_{a}$ is the ambient temperature $\left(T_{a}\right)$ and $v_{p v}$ is the wind velocity $(\mathrm{m} / \mathrm{s})$.

After building the model of the PV system, it is necessary to describe the relationship between solar radiation and the output of the PV system. The beta-distribution probability function can be used to express the output variation of the PV generation system. According to [30]:

$$
f\left(P_{M}\right)=\frac{\Gamma(\alpha+\beta)}{\Gamma(\alpha) \Gamma(\beta)}\left(\frac{P_{M}}{R_{M}}\right)^{\alpha-1}\left(1-\frac{P_{M}}{R_{M}}\right)^{\beta-1}
$$

where $\alpha$ and $\beta$ are the shape parameters of the beta-distribution; $P_{M}$ is the total power of the PV array, and $R_{M}$ is the maximum power that the PV array can output.

(3) Gas Boiler (GB)

The heat required in the IEMG is mainly produced by two devices, a CCHP and a gas boiler. During production, the thermal energy is mainly provided by the CCHP. Once it cannot meet the heating load, the gas boiler (GB) can convert the chemical energy of the fuel into thermal energy with a high conversion efficiency, to achieve the thermal balance of the system. Assuming that the gas boiler converts the energy of the natural gas into heat at a constant conversion efficiency, the thermal power of the natural gas that is consumed by the gas boiler is:

$$
Q_{h, d, D}^{C B}=V_{h, d, D}^{C B} \theta_{N G} / 3.6
$$


and the heat supply efficiency is:

$$
q_{h, d, D}^{C B}=\eta_{d, D}^{G B} Q_{h, d, D}^{C B}
$$

where $Q_{h, d, D}^{C B}$ is the thermal power of the fuel consumed by the boiler $(\mathrm{kW}) ; q_{h, d, D}^{C B}$ is the boiler's heat output $(\mathrm{kW})$; and $\eta_{d, D}^{G B}$ is the heating efficiency coefficient of the boiler.

Similar to the CCHP, some waste heat of the gas boiler can be reused. The formula for the available waste heat efficiency is:

$$
q_{h, d, D}^{C B r}=\eta_{d, D}^{G B r} Q_{h, d, D}^{C B}
$$

where $q_{h, d, D}^{C B r}$ is the total available waste heat of the boiler, and $\eta_{d, D}^{G B r}$ is the waste heat energy efficiency coefficient of the boiler.

(4) Heat Recovery Boiler

The recycling of waste heat is an important means for improving energy efficiency. In IES and IEMGs, a heat recovery boiler (HRB) is used to collect waste heat from the system. The model of the heat recovery boiler is:

$$
q_{h, d}^{r e}=\eta_{r e}\left(q_{s, d, D}^{G A S}+q_{h, d, D}^{C B r}\right)
$$

where $q_{h, d}^{r e}$ is the output of the heat recovery boiler $(\mathrm{kW}), q_{s, d, D}^{G A S}$ is the waste heat from CCHP $(\mathrm{kW})$; $q_{h, d, D}^{C B r}$ is the waste heat from gas boiler $(\mathrm{kW}) ; \eta_{r e}$ is the thermal efficiency of the equipment.

(5) Chiller

There are two kinds of chillers commonly used in IES and IEMGs. These are the absorption chiller (AC) and the electric chiller (EC). ACs are driven by a thermal medium, such as lithium bromide or ammonia solution; during operation, the working medium vaporization absorbs a lot of heat from the refrigerant water, so as to achieve cooling. The refrigeration principle of the EC involves, first, compressing the gas refrigerant by electricity, then discharging the refrigerant into a condenser. Under set pressure and temperature conditions, the low temperature and low pressure refrigerant cools the air or the condensed water in the condenser to achieve a cooling effect.

The models of the two chillers are as follows. For an AC:

$$
q_{h, d}^{A C}=q_{h, d, D}^{A C, i n} \cdot \eta_{d, D}^{A C}
$$

where $q_{s, d}^{A C}$ is the cooling output $(\mathrm{kW}) ; q_{h, d, D}^{A C, i n}$ is the heat input $(\mathrm{kW})$; and $\eta_{d, D}^{A C}$ is the refrigeration coefficient, which is the ratio of the heat input to the cooling output, and it is usually used to measure the performance of an AC. For an EC:

$$
q_{s, d}^{E C}=p_{h, d, D}^{E C, i n} \cdot \eta_{d, D}^{C O P, E C}
$$

where $q_{s, d}^{E C}$ is the cooling output $(\mathrm{kW}) ; p_{h, d, D}^{E C, i n}$ is the electric power input $(\mathrm{kW})$; and $\eta_{d, D}^{C O P, E C}$ is the refrigeration coefficient of the EC.

\subsubsection{Energy Network Model}

The mathematical expression for the energy network, which connects different devices and different regions in the IEMG, can be represented by network topology. Its connection mode is described by the incidence matrix of the topology structure. It consists of the combination of several IES, as shown in Figure 1, with the framework of Figure 2 as an example. As in Figure 1, suppose that the equipment and energy network connection (both from the power grid and the thermal network) in the IEMG are nodes. Each pipe or power line serves as a branch, taking the flow direction of the working medium as the branch direction. A basic model of the energy network expressed by the incidence matrix is by the following. Assume that $\mathrm{V}$ is the node set of the network. and $\mathrm{E}$ is the set of the power lines or pipes (referred to in the following as the set of edges). The energy network can 
be represented by the $\mathrm{v}$ by e incidence matrix $A=\left[A_{i j}\right]$. Thus, each node of the network is a row of the incidence matrix, and each edge is a column [31]. The relationship between the nodes and edges can be indicated by the sign of $A_{i j}$. When $A_{i j}=1$, the node $v_{i}$ is linked with edge $e_{j}$ and the direction points away from $v_{i}$. When $A_{i j}=-1, v_{i}$ is also linked with $e_{j}$ and the direction points towards $v_{i}$. When $A_{i j}=0, v_{i}$ and $e_{j}$ are not linked.

Through this approach, the incidence matrix can represent any connection modes of the network system, as with the energy network. However, in order to describe the energy network more accurately, the incidence matrix needs to be further expanded. Based on the form of the incidence matrix $A$, it is split into the start incidence matrix $A_{1}$ and the end point incidence matrix $A_{2}$, to represent the node set of the starting or ending points of the power and pipe lines, respectively. Therefore, $A_{1}$ and $A_{2}$ are defined as follows:

$$
\begin{gathered}
A_{1}=\left(a_{z k}\right)_{n \times m} \in\{0,1\}^{n \times m} \\
a_{z k}=\left\{\begin{array}{cc}
1, & (z, c)=E_{k} \\
0, & \text { else }
\end{array}\right.
\end{gathered}
$$

and

$$
\begin{gathered}
A_{2}=\left(a_{z k}\right)_{n \times m} \in\{0,-1\}^{n \times m} \\
a_{z k}=\left\{\begin{array}{cc}
-1, & (c, z)=E_{k} \\
0, & \text { else }
\end{array}\right.
\end{gathered}
$$

Hence, suppose that the basic loop set of $G(V, E)$ is $L$ containing $p$ elements, and its basic loop matrix is $B=\left[B_{h k}\right]$. Thus, in the matrix $B$, each element $B_{h k}$ describes the relationship of the loop $L_{h}\left(L_{h} \in L, h=1,2, \ldots, p\right)$ with edge $k$ (a branch of the grid or pipeline in the thermal network). When $B_{h k}=1$, the loop $L_{h}$ is in the same direction as edge $k$; when $B_{h k}=-1$, the loop $L_{h}$ is opposite to the edge $k$. If $B_{h k}=0$, the edge $k$ is not in the loop $L_{h}$. This method can be used to describe most of the network system. The energy network based on the incidence matrix is summarized by [32], and the matrix can express the energy network as:

$$
\left\{\begin{array}{l}
A H=0 \\
B \Delta X=0
\end{array}\right.
$$

where $A$ is the incidence matrix of the energy network, $B$ is the basic loop matrix, $H$ is the energy extensive flow matrix, and $\Delta X$ is the energy-intensive difference matrix. The equivalent transfer characteristics for incompressible fluids in the energy network is:

$$
\begin{gathered}
H=H^{*}=\frac{X_{A 1}-X_{A 2}}{R}, \\
R=\frac{L}{K S}
\end{gathered}
$$

where $H$ is the flow of energy transferred in the network, $H^{*}$ is the equivalent extensive energy flow in the transfer process, $R$ is the transmission resistance, $L$ and $S$ are the length and cross-sectional area of the transmission line, and $K$ is the transfer coefficient of extensibility.

By combining these two equations, the energy transfer characteristic equation set for the energy network can be established in order to describe the energy transfer state at each node. The advantage of the incidence matrix is that it turns the topology relationship, and the structure of the nodes and edges in the network, into variables in a matrix which is convenient for calculations. It is also helpful for making real-time variations of the connection mode during network analysis and, therefore, it simplifies and expands the analysis of the energy network system. In addition, the incidence matrix can be used to calculate the power flow at any position of the energy network, which can improve the accuracy of energy network planning and operation optimization. On the basis of the incidence 
matrix expression for the energy network, models of the electrical network and the thermal network, and their constraints can be determined, as follows.

(1) Electrical Network Model

Based on the above, by taking into account Kirchhoff's current law (KCL), Kirchhoff's voltage law (KVL), and electricity flow constraints for network systems, the power grid model of the IEMG containing the distributed generation sources can be derived.

a. The Kirchhoff's current constraint (KCL):

$$
\sum S_{t}^{E A} f_{t}^{E A}+\sum G_{t}^{E}+r_{t}=d_{t}^{E}
$$

This equation reflects the equilibrium relationship between the inlet current and the outlet current at any node in scenario $t$. In the equation, $S_{t}^{E A}$ is the node-branch incidence matrix of the power grid in scenario $t, f_{t}^{E A}$ is the branch current, $G_{t}^{E}$ is the input power of the node from the power generation equipment, $r_{t}$ is the lost electrical load, and $d_{t}^{E}$ is the electrical load at the node.

b. Kirchhoff's voltage constraint (KVL) and the voltage magnitude constraint are:

$$
\begin{gathered}
Z_{j, t}^{E A} f_{j, t}^{E A}+\left[S_{t}^{E A}\right]_{\text {rowj }}^{T} V_{j, t}=0 \\
V_{\text {min }} \leq V_{j} \leq V_{\text {max }}
\end{gathered}
$$

Here, row $j$ is the $j$-th column, $T$ refers to the transpose, $V$ is the column vector of the node voltage, and $\mathrm{Z}$ is the line impedance. The inequality defines the magnitudes of the node voltage in which $V_{\min }$ and $V_{\max }$ are the maximum and the minimum voltage magnitudes, respectively.

\section{(2) Thermal Network Model}

Similar to the above, using the energy network incidence matrix and Kirchhoff's laws, a model describing the working principles of the thermal network can be established. Three functions are used to define the transmission flow of the working medium, the relationship of heat and flow, and the change of the transmission pressure. In addition, apart from the relationship between the heat transfer and the mass flow, it is necessary to consider the corresponding heat loss [33]. The thermal network model is as follows.

a. The transmission flow constraint:

$$
\sum S_{t}^{H A} q_{t}^{H A}+\sum G_{t}^{H}=d_{t}^{H}
$$

where $S_{t}^{H A}$ is the node-branch incidence matrix of the thermal network in scenario $t, q_{t}^{H A}$ is the energy flow in the branch pipe line in scenario $t, G_{t}^{E}$ is the input power of the node from heat generation equipment, and $d_{t}^{H}$ is the thermal load at the node.

b. The heat-flow constraint:

According to the equivalent energy transfer characteristic equation for the thermal network, the relationship between the available heat and the flow is:

$$
q_{t}^{H A}=\frac{P_{t}}{k\left(T_{A 1}-T_{A 2}\right)}
$$

where $P_{t}$ is the energy (heat) intensity in the pipeline section, $k$ is the specific heat capacity of the working medium, $T_{A 1}$ and $T_{A 2}$ are the feed-water temperature and return-water temperature at the node, respectively. 
c. The transmission pressure constraint:

After the relationship between the heat and the flow in the thermal network has been determined, the heat intensity at the node conforms to the following heat balance constraint:

$$
Z_{j, t}^{H A} q_{j, t}^{H A}+\left[S_{t}^{H A}\right]_{r o w j}^{T} P_{j, t}=0
$$

where $Z_{j, t}^{H A}$ is the demand for the thermal intensity at node $j$ during period $t ; q_{j, t}^{H A}$ is the energy flow at node $j ; P_{j, t}$ is the heat column vector in the pipeline section connected to node $j$.

\subsection{Energy Balance of the IEMG}

From the configuration and structure of the IEMG, as shown in Figure 3, the electrical/ cooling/heating loads should be balanced in any district, or balanced over the whole region, and in all of the different scenarios this principle is basically the same. The energy balance equations in the IEMG are introduced below.

a. Balance of the electrical load (in all scenarios):

$$
p_{s, d}^{S U B}+p_{s, d}^{P V}+p_{s, d}^{C C H P}=l_{s, d}+p_{s, d}^{E C}
$$

The electrical load balance shall be satisfied in any scenario $s(s=c, h, t, e) . p_{s}^{S U B}$ is the power from the external grid, $p_{s, d}^{P V}$ is the PV generation power, $p_{s, d}^{C C H P}$ is the power of the CCHP $(\mathrm{kW}), l_{s, d}$ is the pure electrical load, and $p_{s, d}^{E C}$ is the power of the electric chiller $(\mathrm{kW})$.

b. Balance of the cooling load (in scenarios of a cooling supply period):

The demand for cooling is satisfied by two devices: the electric chiller and the heat adsorption chiller:

$$
q_{h, d}^{C A}=q_{h, d}^{E C}+q_{h, d}^{A C}
$$

where $q_{h, d}^{C A}$ is the total load of cooling, $q_{h, d}^{E C}$ is the input power of the electric chiller, and $q_{h, d}^{A C}$ is the input power of the adsorption chiller.

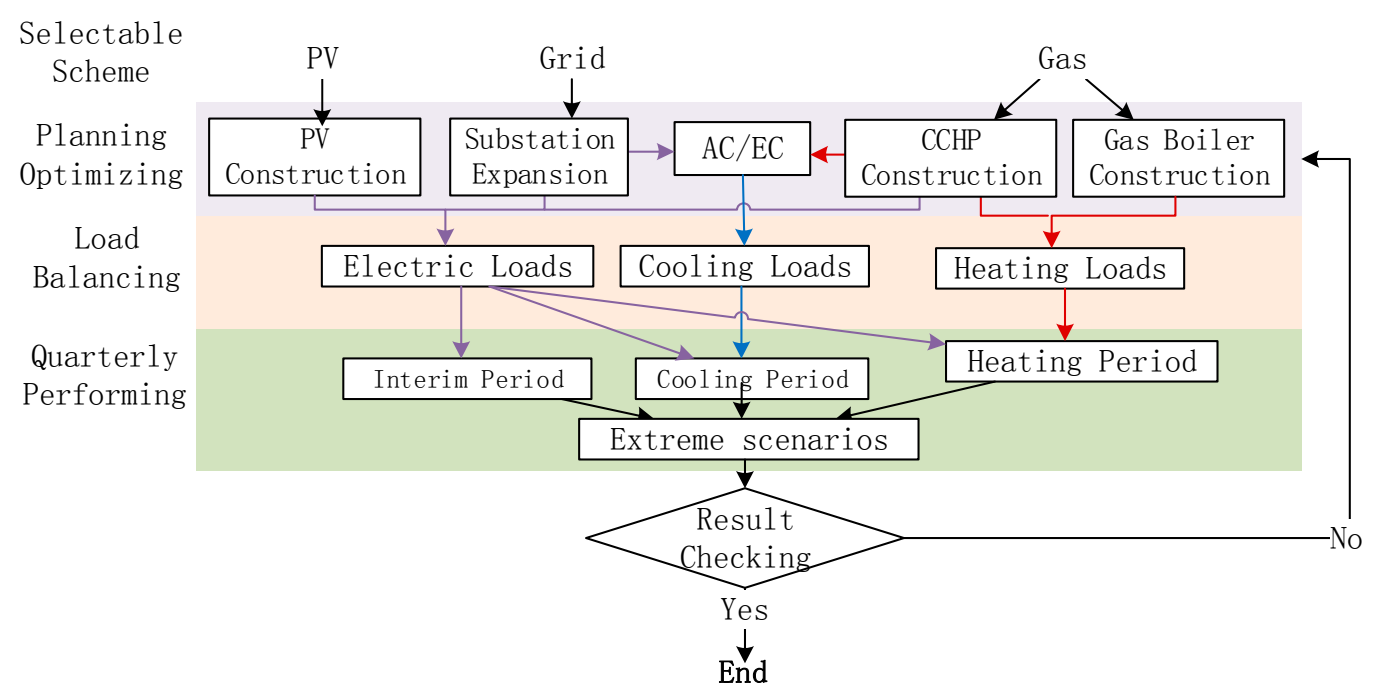

Figure 3. Optimization strategy of IEMG planning. 
c. The balance of heating load (in the scenario of a heating supply period):

The heating load is satisfied by the CCHP and the gas boiler:

$$
q_{h, d}^{H}=q_{h, d}^{C C H P}+q_{h, d}^{G B}
$$

where $q_{h, d}^{H}$ is the total heating load, $q_{h, d}^{\mathrm{CCHP}}$ is the heat supplied by the CCHP, and $q_{h, d}^{G B}$ is the heat from the gas boiler.

\section{The IEMG Planning Optimization Model}

\subsection{Planning Process and Framework}

In this study, the procedure for IEMG planning is summarized as follows:

(1) Extract the regional division, loads, and other planning-related data and information, and carry out the overall regional energy supply equipment configuration (for electricity, heating, and cooling).

(2) Obtain the overall configuration capacity of the energy supply equipment from step 1, combined with the load characteristics of each region, and do the regional equipment type selection and capacity optimization.

(3) According to the equipment selection and capacity optimizing results, deduce the electricity, heating and the cooling load balance operation simulation of each region, and output the results.

(4) Deduce the load balance operation simulation on the basis of quarterly and extreme scenarios, and output the results.

(5) Test and determine whether the regional and quarterly simulation results conform to the energy flow and all other constraints.

(6) If the result does not satisfy all the constraints, adjust the selection and capacity results until all constraints are met, then output the corresponding configuration.

In the steps above, Step (1) is preparatory work. Its main function is to determine the macroscopic capacity of the whole region on the basis of the known information, in order to narrow the scope of subsequent optimization. Step (2) determines the equipment selection and the installed capacity of each region on the basis of the macro-planning results. Steps (3) and (4) formulate the operation strategy and calculate the system operation cost. This is done through regional and situational operation simulation, using the planning scheme and the scenarios determined in the previous steps. Steps (5) and (6) ensure that the results meet the requirements of the constraints, and improve the accuracy of the optimization. The process is also shown in Figure 3.

Assume that an IEMG satisfies its electrical/heating/cooling loads through PV panels, natural gas (for the CCHP and gas boiler), as well as by purchasing electricity from the external grid. It is then necessary to consider the IEMG plan from the aspect of expanding its original capacity or building a new transformer substation and PV system to supply electricity. A new CCHP construction can meet the electrical/cooling/heating energy demands. Increasing the number of gas boilers compensates for heating between the CCHP heating output and the heating demand. Adding more chilling equipment can satisfy the cooling load. Accordingly, the decision process of the IEMG planning model is given in Figure 4.

Thus, the decision variables in the model can be classified into two types: construction and operation.

The constructional decision variables are mainly binary, where ' 0 ' and ' 1 ' mean to undo/do a decision, respectively. To be specific, in the type selection option $d, x_{d, D}^{C C H P}$ is the decision variable for whether to invest in the new CCHP in district $D$ or not; similarly, $x_{d, D}^{G E}$ is the decision variable of any other power generator in district $D . x_{d, D}^{G H}$ is the decision variable of the heating generator, $x_{d, D}^{G C}$ is the 
decision variable of the cooling generator, and $x_{J}^{S U B}$ is the decision variable of a new or expanded transformer substation.

The operation decision variables are continuous and include: the electrical generation output of the CCHP, $g_{s}^{\mathrm{CCHP}}$; the heating generation output of the CCHP, $q_{s}^{\mathrm{CCHP}}$; the heating power of the gas boiler, $q_{s}^{G B}$; the power of the electricity purchased from the external gird to the substation, $g_{s}^{S U B}$; and the power of the cooling generation equipment, $q_{s}^{C}$.

In addition, there are four typical load periods mentioned during the optimization, which measure the economics of the operational strategies. These are the transitional period $(s=t)$, the cooling supply period $(s=c)$, the heating supply period $(s=h)$, and the extreme period $(s=e)$. The extreme period indicates the unusual and sudden situation in which high cooling supplementation is required in summer, and makes sure that the results of the planning are reliable under extreme conditions.

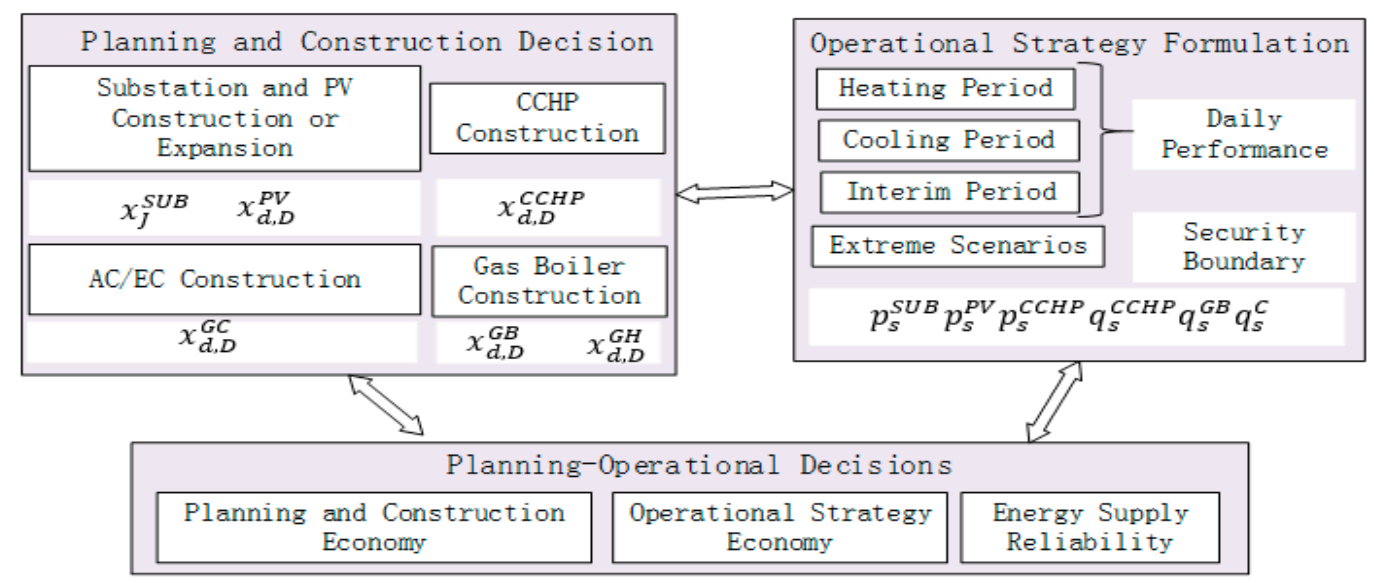

Figure 4. The main variables and decisions of the IEMG planning model.

\subsection{The Objective Function}

The overall objective of the planning model is to meet the maximum energy needs of the whole region while minimizing the sum of the construction cost and the operation cost. Hence, the objective function in this model consists of three parts: the planned construction cost, the planned operation cost, and the value of lost loads,

$$
\min C_{I N V}+C_{O P E}+C_{V O L L}
$$

where $C_{I N V}$ is the planned construction cost, $C_{O P E}$ is the planned operation cost, and $C_{V O L L}$ is the value of lost load. The calculation of each part is as follows.

(1) The planned construction cost:

The function of the planning model is to select the economic optimal among several construction schemes, then it should be noticed that there are many different supplement construction portfolios. The equation below gives the cost which is determined for construction. Now, assume that plan $D$ is known to be executed, thus, the planned construction cost $C_{I N V}$ of plan D includes the construction cost of the CCHP, the construction cost of the electrical/cooling/heating generator, and the expansion cost of the transformer substation:

$$
\begin{gathered}
C_{I N V}=\sum_{d \in \Omega} \sum_{D \in \phi_{d}^{C C H P}} M_{d, D}^{P V} x_{d, D}^{P V}+\sum_{J \in \phi^{\text {SUB }}} M_{J}^{S U B} x_{J}^{S U B}+\sum_{d \in \Omega} \sum_{D \in \phi_{d}^{C C H P}} M_{d, D}^{C C H P} x_{d, D}^{C C H P} \\
+\sum_{d \in \Omega} \sum_{D \in \phi_{d}^{C C H P}} M_{d, D}^{G B} x_{d, D}^{G B}+\sum_{d \in \Omega} \sum_{D \in \phi_{d}^{C C H P}} M_{d, D}^{G H} x_{d, D}^{G H}+\sum_{d \in \Omega} \sum_{D \in \phi_{d}^{C C H P}} M_{d, D}^{G C} x_{d, D}^{G C}
\end{gathered}
$$

where $M_{d, D}^{P V}, M_{d, D}^{C C H P}, M_{d, D}^{G B}, M_{d, D}^{G H}$, and $M_{d, D}^{G C}$ are the construction costs of PV panels, the CCHP, the gas boiler, the heating equipment, and the cooling equipment, respectively, in region $d . M_{J}^{S U B}$ is the cost 
of expanding the transformer substation ( $J$ represents plan $J$ ); and $x$ is a binary decision variable, where the cost is taken into account when the value is 1 .

(2) The planned operation cost:

Here, equipment maintenance and depreciation costs are put aside. The planned operation cost $C_{O P E}$ includes the cost of the fuel for the CCHP and the gas boiler, and the cost of purchased electricity:

$$
C_{O P E}=\sum_{r} \frac{r}{(1+i)^{r}} \sum_{d \in \Omega} \sum_{s=c, h, t} \varepsilon_{s}\left(\operatorname{Pr}^{G A S} F_{s, d}^{f u e l}+\operatorname{Pr}^{S U B} p_{s, d}^{S U B}\right)
$$

where $r$ represents the system run cycle, $\sum_{r} \frac{r}{(1+i)^{r}}$ is the total net cost of the annual operation, $i$ is the discount rate, and $\varepsilon_{s}$ is the proportional contribution of scenario $s$ to the entire planning period. For instance, when the planning period is one year (12 months), if the heating supply period contains four months from 15 November to 15 March, the proportion is $4 / 12=0.333$; if the cooling supply period contains three months from 15 June to 15 September, the proportion is $3 / 12=0.25$, then the transitional period contains the other five months and the proportion is 5/12 $=0.417 . \mathrm{Pr}^{G A S}$ and $\mathrm{Pr}^{S U B}$ are the prices of natural gas and external electricity, respectively. $F_{s, d}^{f u e l}$ is the fuel consumption per unit time in district $d$, which consists of the fuel consumed by the CCHP and the gas boiler:

$$
F_{s, d}^{f u e l}=\sum_{D \in \Phi_{d}^{C C H P}} F_{s, d, D}^{C C H P}+\sum_{D \in \Phi_{d}^{G B}} F_{s, d, D}^{G B} .
$$

$g_{s, d}^{S U B}$ is the quantity of the electricity purchased from the external grid by a substation in district $d$.

(3) The value of the lost load:

In this part, $C_{V O L L}$ indicates the compensation cost for unsatisfied electrical/heating/cooling loads, which are not supplied during scenario $s$ in district $d$.

$$
C_{V O L L}=P^{V O L L} \sum_{d \in \Omega} \sum_{s} R_{d, s}, \quad s=c, h, t, e
$$

Here, $R_{d, s}$ is the capacity of the lost loads and $P^{V O L L}$ is the cost coefficient of the lost loads. It should be pointed out that $P^{V O L L}$ is set to a relatively high value, in order to avoid load loss during operation.

\subsection{Constraint Conditions}

In planning the IEMG, the variation of generation equipment parameters in the model should be within a certain range. Their constraint conditions are given in the following.

a. The permeability constraint on the distributed generation (DG):

The proportion of the DG to the total installed capacity should be within a certain range:

$$
\begin{gathered}
p_{d, D}^{P V, \text { min }} \leq p_{d, D}^{P V} \leq p_{d, D}^{p v, \text { max }} \\
v_{d, D}^{P V, \text { min }} \leq v_{d, D}^{P V} \leq v_{d, D}^{P V, \text { max }} \\
N_{d, D}^{P V, \text { min }} \leq \mathrm{NG}_{d, D}^{P V} \leq N_{d, D}^{P V, \text { max }}
\end{gathered}
$$

where in the plan $D$ for region $d, p_{d, D}^{P V, \text { min }}$ and $p_{d, D}^{p v, \text { max }}$ are the lower and upper limits of the active power of the DG $(\mathrm{kW}) . v_{d, D}^{P V, \text { min }}$ and $v_{d, D}^{P V, \text { max }}$ are the lower and upper limits of the reactive power $(\mathrm{kW})$ of DG, respectively. $p_{d, D}^{P V}$ and $v_{d, D}^{P V}$ are the actual active power and reactive power of DG in district $d(\mathrm{~kW})$. $N_{d, D}^{P V, \text { min }}$ and $N_{d, D}^{P V, \text { max }}$ are the lower and upper limits of the number of the DG in the system. $\mathrm{NG}_{d, D}^{P V}$ is the number of DGs in district $d$. 
b. Operational constraints for the CCHP:

The operational constraints for the CCHP include the maximum and the minimum outputs, the ramp rate, and the maximum and the minimum continuous running times. They are given by:

$$
\sum_{D \in \phi_{d}^{C C H P}} x_{d, D}^{C C H P} p_{m i n, d, D}^{C C H P} \leq p_{d, D}^{C C H P} \leq \sum_{D \in \phi_{d}^{\mathrm{CCHP}}} x_{d, D}^{C C H P} p_{m a x, d, D}^{C C H P}
$$

where $p_{\min , d, D}^{C C H P}$ and $p_{\max , d, D}^{C C H P}$ are the lower and the upper limits of the CCHP's output (kW).

The number of generation units in each district needs to be limited, due to geographical factors. In this model, we only limit the total number of CCHP units, and allow one per district:

$$
\sum_{D \in \phi_{d}^{C C H P}} X_{d, D}^{C C H P} \leq 1
$$

where $X_{d, D}^{C C H P}$ is the total number of CCHP generation units in district $d$. This constraint can be adjusted according to planning requirements.

c. Operation constraints on the gas boiler:

The output heating power of the gas boiler during operation should be no larger than its rated power:

$$
0 \leq q_{h, D}^{G B} \leq \sum_{D \in \phi_{d}^{G B}} x_{d, D}^{G B} q_{\max , d, D}^{G B}
$$

where $q_{\max , d, D}^{G B}$ is the rated power.

Similarly, the construction constraint for the gas boiler is:

$$
\sum_{D \in \Phi_{d}^{G B}} X_{d, D}^{G B} \leq 1
$$

This ensures that the boilers in district $d$ are of the same capacity.

d. Constraints on the chillers:

The power of the adsorption chiller and the electrical chiller during operation should be no larger than their rated power:

$$
0 \leq q_{s, d}^{A C / E C} \leq q_{\max , s, d}^{A C / E C}
$$

where $q_{\max , s, d}^{A C / E C}$ is the rated power of the chiller $(\mathrm{kW})$.

e. The power flow constraint:

The power flow of the system should be limited according to the magnitude of the current in the electrical network:

$$
\left|f^{E A}\right| \leq y^{E A} f_{\max }^{E A}
$$

where $f_{\max }^{E A}$ is the upper limit of the current magnitude and $y^{E A}$ is the conductance value at the corresponding node.

f. The balance constraint for heat loss in the thermal network:

If there is too much heat loss in the pipelines of the thermal network, the temperature of the working medium in the pipelines will become lower than the temperature of the working medium in the return-water system. As a result, the thermal network will be ineffective. To ensure the efficiency of the thermal network, we therefore need to ensure that the power (temperature) of the usable heat in 
the pipelines is higher than a critical value and lower than the maximum power that can be transferred in the pipelines:

$$
P_{t}^{\min } \leq P_{i, t}^{*} \leq P_{t}^{\max }
$$

where $P_{i, t}{ }^{*}$ is the power of the usable heat in the working medium at node $j, P_{t}^{\min }$ is the lower critical value of the power of the usable heat, and $P_{t}^{\max }$ is the maximum power of the usable heat. If the working medium flows away from node $i$ then the value of $P_{i, t}{ }^{*}$ is positive, otherwise it is negative.

g. The supply capacity constraint for the transformer substation (in the external grid):

In the transformer substation, the total power supply capacity should not be greater than the product of the load and the capacity-load ratio, expressed as:

$$
\begin{aligned}
& \gamma_{\min } p_{s}^{S U B} \leq p_{0}^{S U B}+\sum_{J \epsilon \varnothing^{S U B}} x_{J}^{S U B} p_{J}^{S U B} \leq \gamma_{\max } p_{s}^{S U B} \\
& \sum_{J \in \varnothing^{S U B}} x_{J}^{S U B} \leq 1
\end{aligned}
$$

The first inequality describes the relationship between the original power supply capacity $p_{0}^{S U B}$

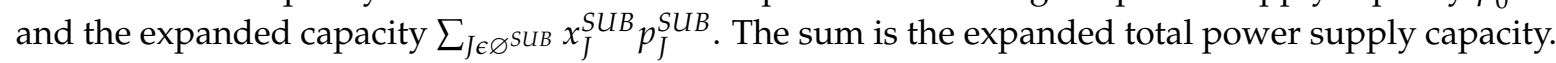
To ensure accuracy and effectiveness in planning, the product of the total power supply capacity and capacity-load ratio $\gamma$ should be valid for the extreme load scenario $(s=e)$. The range of the capacity-load ratio $\gamma$ in this model is $1.8 \sim 2.1$. The second inequality means that only one transformer substation expansion plan in the set $\varnothing^{S U B}$ will be carried out.

\subsection{Calculation Method}

Generally, dynamic programming (DP) algorithms could be implemented to energy planning optimization [34]. Here, we used the mixed integer linear programming (MILP) method to solve the IEMG planning model. The model involves the following decision variables: the output of the PV power system, the input and outputs of the CCHP, the electricity purchased from or sold to the external power grid, the input of the conversion equipment, and the input and output of the gas boiler. The model can be solved using a mature algorithm, or directly by commercial software, such as CPLEX, GUROBI, and LINGO [35]. In this study, the model was built by the software MatLab and Yalmip, and solved by the optimization software GUROBI. The process of the optimization algorithm is as in Figure 5.

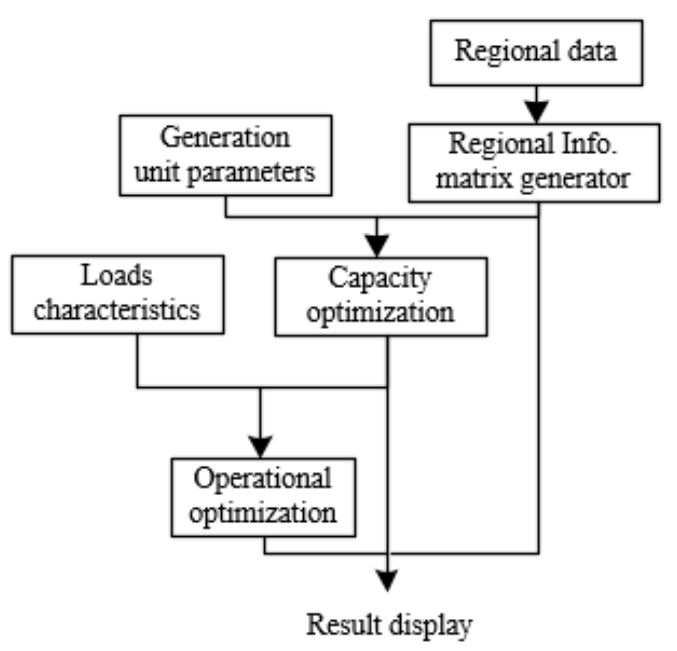

Figure 5. The optimization algorithm process of IEMG planning. 


\section{Case Study}

\subsection{Case Description}

In this work, a new development area of a municipality was taken as a case study. The planning data and the predicted annual saturated electrical/cooling/heating load data were already known, as shown in Figure 6.

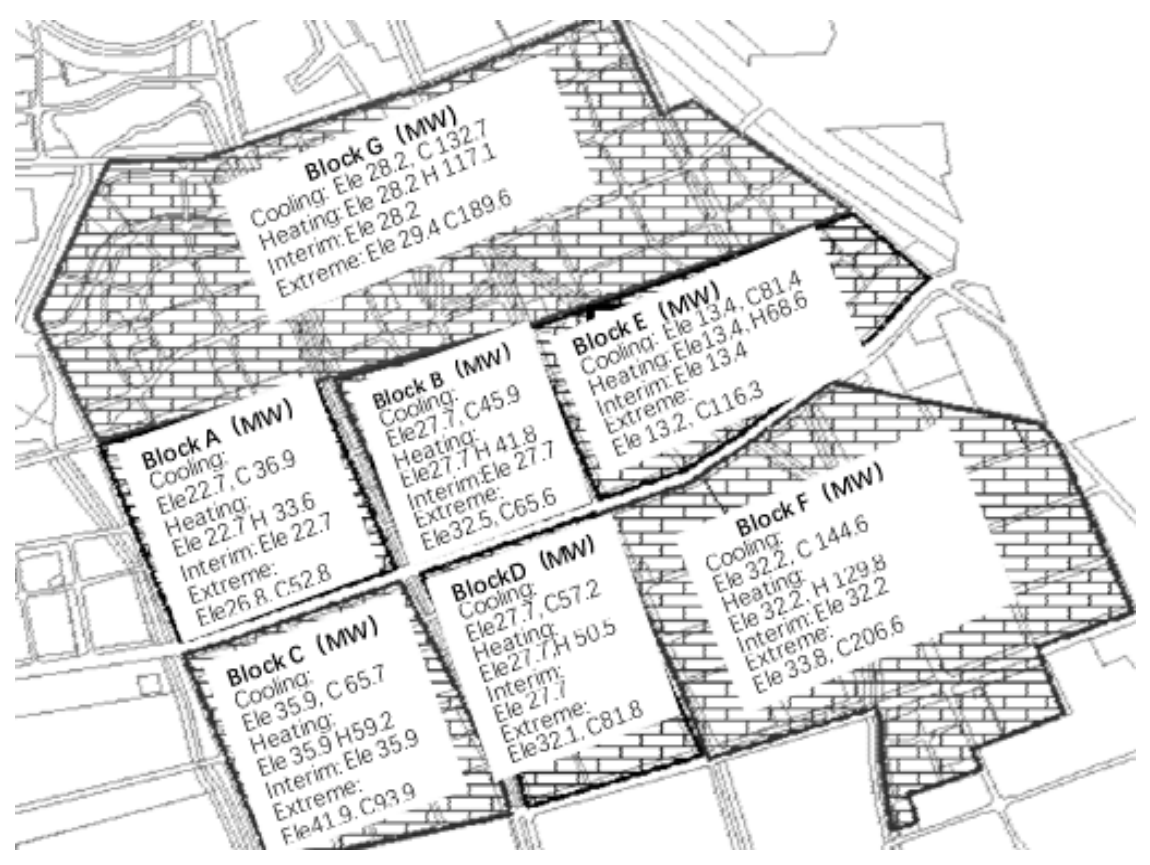

Figure 6. Map showing the energy supply planning information of the case study area.

From Figure 5, the data of the loads were classified under four scenarios: cooling period, heating period, transitional period, and extreme cooling period. It should be noted that during the calculation the load on the air conditioner should be subtracted during the cooling period.

Alternative planning options for the energy supply in the region are listed in Table 1. The parameters of the different CCHP units are listed in Table 2. The parameters used for the 9.5 MW $\mathrm{CCHP}$ unit are the same as those for the $5 \mathrm{MW}$ unit.

Table 1. Alternative planning options for the energy supply equipment. Combined cooling, heating, and power unit (CCHP), photovoltaic (PV), absorption chiller (AC), electric chiller (EC), heat recovery boiler (HRB); USD: US dollars.

\begin{tabular}{|c|c|c|c|c|c|}
\hline \multicolumn{3}{|c|}{ Substation Expansion } & \multicolumn{3}{|c|}{ СCHP } \\
\hline Scheme No. & Capacity (MVA) & Construction Cost & Scheme No. & Capacity (MW) & Construction Cost \\
\hline 1 & $1 \times 50$ & 125 & 1 & 1 & 185 \\
\hline 2 & $2 \times 50$ & 250 & 2 & 2 & 375 \\
\hline 3 & $3 \times 50$ & 375 & 3 & 3 & 550 \\
\hline 4 & $4 \times 50$ & 500 & 4 & 5 & 850 \\
\hline 5 & $5 \times 50$ & 625 & 5 & 9.5 & 1600 \\
\hline 6 & $6 \times 50$ & 750 & & & \\
\hline \multicolumn{3}{|c|}{ Gas Boiler } & \multicolumn{3}{|c|}{ Others } \\
\hline Scheme No. & Alternative Options (MW) & Construction Cost & Equipment & Construction Cost & Unit \\
\hline 1 & 50 & 440 & PV & 130 & $10^{4} \mathrm{USD} / \mathrm{MW}$ \\
\hline 2 & 100 & 900 & $\mathrm{AC}$ & 10 & $10^{4} \mathrm{USD} / \mathrm{MW}$ \\
\hline 3 & 150 & 1350 & $\mathrm{EC}$ & 15 & $10^{4} \mathrm{USD} / \mathrm{MW}$ \\
\hline 4 & 200 & 1750 & HRB & 2.5 & $10^{4} \mathrm{USD} / \mathrm{MW}$ \\
\hline
\end{tabular}


Table 2. Performance parameter of the CCHP.

\begin{tabular}{ccccccc}
\hline \multirow{2}{*}{ Full Capacity (MW) } & \multicolumn{6}{c}{ Characteristic Function Coefficient } \\
\cline { 2 - 7 } & $\alpha^{G E}$ & $\beta^{G E}$ & $\alpha^{G A S}$ & $\beta^{G E}$ & $\alpha^{W A}$ & $\beta^{W A}$ \\
\hline 1 & 0.421 & -222.411 & 0.211 & 3.624 & 0.149 & 81.731 \\
2 & 0.466 & -657.422 & 0.219 & 13.644 & 0.151 & 90.737 \\
3 & 0.479 & -758.940 & 0.208 & 94.383 & 0.153 & 173.562 \\
5 & 0.472 & -896.264 & 0.207 & 125.832 & 0.149 & 204.828 \\
9.5 & 0.470 & -915.262 & 0.204 & 130.261 & 0.146 & 220.152 \\
\hline
\end{tabular}

Other operational parameters included the calorific value and price of gas: $32.967 \mathrm{MJ} / \mathrm{m}^{3}$ and $0.5 \mathrm{USD} / \mathrm{m}^{3}$, respectively; the external electricity price in this area was a commercial price, which was $0.16 \mathrm{USD} / \mathrm{kWh}$ (the purchase price from the weighted average of the peak-valley electricity prices). The total planning period was 10 years and the annual discount rate $i$ was 5\%. To minimize the lost load, the value of $P^{V O L L}$ was set to $150,000 \times 10^{4} \mathrm{USD} / \mathrm{MW}$.

Four cases were set to make the calculation of the planning more accurate:

CASE 1 Electricity is only purchased from the external power grid, without consideration of PV and CCHP;

CASE 2 A 4 MW PV system at least is built in each district and electricity can be purchased from the external power grid, without consideration of the CCHP;

CASE 3 CCHP construction is considered and electricity can be purchased from the external power grid when the output of the CCHP is insufficient, without consideration of PV;

CASE 4 A 4 MW PV generation system at least is built in each district, a CCHP is considered, and electricity can be purchased from the external power grid when the outputs of the CCHP and the PV are insufficient.

\subsection{Results and Analysis}

The IEMG planning result for each region as in Figure 7, and the overall economic results are shown in Table 3.

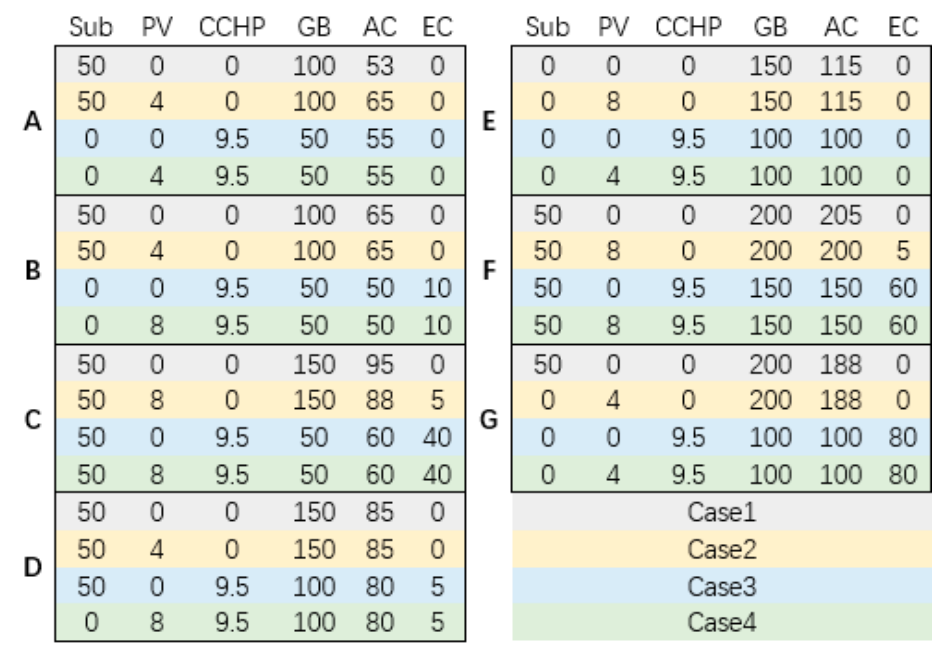

Figure 7. The IEMG planning result of each region in different cases (Unit: MW). 
Table 3. Cost comparison of the different case study scenarios.

\begin{tabular}{cccccc}
\hline \multicolumn{2}{c}{ Comparative Case } & CASE 1 & CASE 2 & CASE 3 & CASE 4 \\
\hline $\begin{array}{c}\text { Selected } \\
\text { Scheme }\end{array}$ & & No PV & With PV & No PV & PV \\
No CCHP & With CCHP & And CCHP \\
\hline & PV & 0 & 3718.75 & 0 & 3718.75 \\
Construction Cost & 0 & 0 & $11,221.875$ & $11,221.875$ \\
& GB\&HRB & $11,161.72$ & $11,161.72$ & 6696.40625 & 6696.40625 \\
& Substation & 750 & 625 & 375 & 250 \\
& AC/EC & 7496.41 & 7496.41 & 6591.40625 & 6591.40625 \\
& In Total & $19,408.13$ & $23,001.88$ & $24,884.6875$ & $28,478.4375$ \\
\hline \multirow{5}{*}{ Operational Cost } & PV & 0 & $42,276.42$ & 0 & $42,276.42$ \\
& Curchased electricity & $322,498.77$ & $257,164.66$ & $206,985.31$ & $160,298.32$ \\
& GB\&HRB & $53,500.78$ & $53,500.78$ & $44,561.415$ & $44,561.41$ \\
& In Total & $375,999.53$ & $352,941.86$ & $351,288.91$ & $340,731.23$ \\
\hline \multicolumn{7}{c}{ Total Cost } & $395,407.66$ & $395,407.66$ & $375,943.73$ & $376,173.59$ \\
\hline \multicolumn{7}{c}{} & (unit: $10^{4}$ USD). & & &
\end{tabular}

From Figure 7 and Table 3, the results showed that: in CASE 1, the capacity of the transformer substation is $6 \times 50 \mathrm{MVA}$, the construction cost is 0.194 billion USD, and the total planning cost, including the operation cost, is 3.954 billion USD. In CASE 2, a 4 MW PV generation source is constructed in each of the seven districts, providing $28 \mathrm{MW}$ in total, and the capacity of the substation is $5 \times 50 \mathrm{MVA}$, the construction cost is 0.230 billion USD, and the total planning cost is 3.954 billion USD. In CASE 3, a 9.5 MW CCHP is constructed in each district (66.5 MW in total), the capacity of the substation is $3 \times 50 \mathrm{MVA}$, the construction cost is 0.249 billion USD, and the total planning cost is 3.759 billion USD. In CASE 4, a 4 MW PV source and a 9.5 MW CCHP are constructed in each district, the capacity of the substation is $2 \times 50 \mathrm{MVA}$, the construction cost is 0.285 billion USD, and the total planning cost is 3.762 billion USD.

In CASE 1, the demands for electricity are satisfied by purchasing electricity from the external power grid and heating is from the gas boiler. This scheme involved the least equipment. The system's structure was relatively simple, and the construction cost was, therefore, the lowest, but the energy supply form was simple and the operational cost was relatively high: 3.225 billion and 0.535 billion USD for electricity and heat, respectively, and 3.760 billion USD in total (which is the highest of the four CASES). In CASE 2, due to PV sources in each district (28 MW in total), the construction cost of the whole system was increased by $18.52 \%$ compared to CASE 1 . However, the operation cost of the external electricity was lower, and the thermal generation's cost remained unchanged, leading to CASE 2 having a $5.31 \%$ reduction in operation cost and a $4.13 \%$ decrease in total planning costs. In CASE 3, a CCHP system was added to supply electricity and thermal energy. Compared to CASE 1, the construction cost was $28.22 \%$ higher, but due to the application of the CCHP the cost of purchasing electricity was $31.71 \%$ lower. The cost of the heating supply was $16.70 \%$ lower, and thus the total cost decreased by $4.86 \%$. In CASE 4 , both PV and CCHP are constructed, which meant that the scheme combines the characteristics of CASES 2 and 3. Therefore, compared to CASE 1, the construction cost was $46.73 \%$, higher but the operation cost decreased by $9.37 \%$. Lower operating costs offset the higher construction costs, making CASE 4 the lowest costing among the four cases. Therefore, in CASES 2, 3 , and 4 , the total planning-operation costs were decreased by $3.74 \%, 4.86 \%$, and $6.63 \%$ compared to CASE 1, respectively. To sum up, the application of PV and the CCHP in the IEMG is efficient in reducing the total planning-operation cost as a whole. 


\section{Conclusions}

This paper presents an IEMG planning model with distributed photovoltaic by MILP. First of all, the model determines the capacity construction configuration of the energy production equipment by known electricity, heating and cooling loads. Second, to further improve the feasibility of the planning results, the calculated capacity allocation is put into operational cost analysis of heating, cooling, transitional and extreme load scenarios. The model takes district energy suppliers as the main investors; the optimized capacity configuration can meet the overall energy demand of the region in different scenarios and, at the same time, give the construction and operation cost of different sub-regions. A case study is given to prove the validity of the model. The case study is in a seven sub-district development zone, and four comparison schemes are given: CASE 1 (electricity supplied by an external power grid), CASE 2 (supplied by an external grid and PV), CASE 3 (supplied by an external grid and CCHP), and CASE 4 (supplied by all above equipment). The calculations show that the ranking of the total costs is CASE $1>$ CASE 2 > CASE $3>$ CASE 4 . Compared to CASE 1 , the total planning-operation costs in the other three cases are decreased by $3.74 \%, 4.86 \%$, and $6.63 \%$, respectively, which reflects the fact that the construction of the distributed PV and CCHP generation sources are beneficial for reducing the total planning-operation costs. From the results of the model calculation, the model we have proposed can be seen as a theoretical reference for the planning of multi-district IES (an IEMG in this paper).

The study could be further improved in the following aspects: the electrical network model and the thermal network model are relatively simple, and only the constraints of the power flow in the power grid and the thermal network are discussed, without consideration of the variation of energy quantity flow rate, the variations of temperature and pressure in the thermal network, or time delay in the thermal network's heat transmission. In addition, the planning and construction of the framework of the energy network is not fully investigated.

Author Contributions: Methodology, H.H. and Z.T.; Writing-Original Draft Preparation, H.H.; Writing-Review and Editing, all the authors.

Funding: This research was funded by National Natural Science Foundation of China under Grant No. 71774039.

Conflicts of Interest: The authors declare no conflict of interest.

\section{References}

1. Mei, S.; Li, R.; Xue, X.; Chen, Y.; Lu, Q.; Chen, X.; Carsten, D.A.; Li, R.; Chen, L. Paving the Way to Smart Micro Energy Grid: Concepts, Design Principles, and Engineering Practices. CSEE J. Power Energy Syst. 2017, 3, 440-449. [CrossRef]

2. Lund, H.; Münster, E. Integrated energy systems and local energy markets. Energy Policy 2006, 34, 1152-1160. [CrossRef]

3. Wang, Y.; Huang, Y.; Wang, Y.; Yu, H.; Li, R.; Song, S. Energy Management for Smart Multi-Energy Complementary Micro-Grid in the Presence of Demand Response. Energies 2018, 11, 974. [CrossRef]

4. Ming, N.; Wei, H.; Guo, J.; Su, L. Research on Economic Operation of Grid-Connected Micro-grid. Power Syst. Technol. 2010, 34, 38-42.

5. Han, L.; Wang, F.; Tian, C. Economic Evaluation of Actively Consuming Wind Power for an Integrated Energy System Based on Game Theory. Energies 2018, 11, 1476. [CrossRef]

6. Chicco, G.; Mancarella, P. Distributed Multi-generation: A Comprehensive View. Renew. Sustain. Energy Rev. 2009, 13, 535-551. [CrossRef]

7. Liu, X.; Wang, S.; Sun, J. Energy Management for Community Energy Network with CHP Based on Cooperative Game. Energies 2018, 11, 1066. [CrossRef]

8. Liu, X.Z.; Wu, J.Z.; Jenkins, N.; Bagdanavicius, A. Combined Analysis of Electricity and Heat Networks. Appl. Energy 2016, 162, 1238-1250. [CrossRef] 
9. Pan, Z.G.; Guo, Q.L.; Sun, H.B. Interactions of District Electricity and Heating Systems Considering Time-scale Characteristics Based on Quasi-Steady Multi-Energy Flow. Appl. Energy 2016, 167, 230-243. [CrossRef]

10. Zeng, Q.; Fang, J.K.; Li, J.H.; Chen, Z. Steady-state Analysis of the Integrated Natural Gas and Electric Power System with Bi-directional Energy Conversion. Appl. Energy 2016, 184, 1483-1492. [CrossRef]

11. Wang, W.L.; Wang, D.; Jia, H.J.; Chen, Z.Y.; Guo, B.Q.; Zhou, H.M.; Fang, M.W. Steady State Analysis of Electricity-gas Regional Integrated Energy System with Consideration of NGS Networks Status. Proc. CSEE 2017, 37, 1293-1304.

12. Shabanpour-Haghighi, A.; Seifi, A.R. An Integrated Steady-state Operation Assessment of Electrical, Natural Gas and District Heating Networks. IEEE Trans. Power Syst. 2016, 31, 3636-3647. [CrossRef]

13. Wang, Y.R.; Zeng, B.; Guo, J.; Shi, J.Q.; Zhang, J.H. Multi-energy Flow Calculation Method for Integrated Energy System Containing Electricity, Heat and Gas. Power Syst. Technol. 2016, 40, 2942-2950.

14. Wang, R.; Gu, W.; Wu, Z. Economic and Optimal Operation of Combined Heat and Power Microgrid with Renewable Energy Resources. Autom. Electr. Power Syst. 2011, 35, 22-27.

15. Liu, X.; Wu, H. A Control Strategy Operation Optimization of Combine Cooling Heating and Power System Considering Solar Comprehensive Utilization. Autom. Electr. Power Syst. 2015, 39, 1-6. [CrossRef]

16. Wang, Y.; Yu, H.; Yong, M.; Huang, Y.; Zhang, F.; Wang, X. Optimal Scheduling of Integrated Energy Systems with Combined Heat and Power Generation, Photovoltaic and Energy Storage Considering Battery Lifetime Loss. Energies 2018, 11, 1676. [CrossRef]

17. Zhou, X.; Guo, C.; Wang, Y.; Li, W. Optimal Expansion Co-planning of Reconfigurable Electricity and Natural Gas Distribution Systems Incorporating Energy Hubs. Energies 2017, 10, 124. [CrossRef]

18. Unsihuay-Vila, C.; Marangon-Lima, J.W.; de Souza, A.Z.; Perez-Arriaga, I.J.; Balestrassi, P.P. A Model to Long-term, Multiarea, Multistage, and Integrated Expansion Planning of Electricity and Natural Gas Systems. IEEE Trans. Power Syst. 2010, 25, 1154-1168. [CrossRef]

19. Saldarriaga, C.A.; Hincapié, R.A.; Salazar, H. A Holistic Approach for Planning Natural Gas and Electricity Distribution Networks. IEEE Trans. Power Syst. 2013, 28, 4052-4063. [CrossRef]

20. Qiu, J.; Dong, Z.Y.; Zhao, J.H.; Xu, Y.; Zheng, Y.; Li, C.; Wong, K.P. Multi-stage Flexible Expansion Co-planning Under Uncertainties in a Combined Electricity and Gas Market. IEEE Trans. Power Syst. 2015, 30, 2119-2129. [CrossRef]

21. Barati, F.; Seifi, H.; Sepasian, M.S.; Nateghi, A.; Shafie-khah, M.; Catalao, J.P.S. Multi-period Integrated Framework of Generation, Transmission, and Natural Gas Grid Expansion Planning for Large-scale Systems. IEEE Trans. Power Syst. 2015, 30, 2527-2537. [CrossRef]

22. Qiu, J.; Dong, Z.Y.; Zhao, J.H.; Meng, K.; Zheng, Y.; Hill, D.J. Low Carbon Oriented Expansion Planning of Integrated Gas and Power Systems. IEEE Trans. Power Syst. 2015, 30, 1035-1046. [CrossRef]

23. Shao, C.; Shahidehpour, M.; Wang, X.; Wang, X.; Wang, B. Integrated Planning of Electricity and Natural Gas Transportation Systems for Enhancing the Power Grid Resilience. IEEE Trans. Power Syst. 2017, 32, 4418-4429. [CrossRef]

24. Zhao, B.; Conejo, A.J.; Sioshansi, R. Coordinated Expansion Planning of Natural Gas and Electric Power Systems. IEEE Trans. Power Syst. 2017, 33, 3064-3075. [CrossRef]

25. Odetayo, B.; MacCormack, J.; Rosehart, W.D.; Zareipour, H. A Sequential Planning Approach for Distributed Generation and Natural Gas Networks. Energy 2017, 127, 428-437. [CrossRef]

26. Khaligh, V.; Buygi, M.; Anvari-Moghaddam, A.; Guerrero, M. A Multi-Attribute Expansion Planning Model for Integrated Gas-Electricity System. Energies 2018, 11, 2573. [CrossRef]

27. Li, G.; Wang, R.; Zhang, T.; Ming, M.; Sciubba, E. Multi-Objective Optimal Design of Renewable Energy Integrated CCHP System Using PICEA-g. Energies 2018, 11, 743. [CrossRef]

28. Wu, D.; Wang, R. Combined Cooling, Heating and Power: A Review. Prog. Energy Combust. Sci. 2006, 32, 459-495. [CrossRef]

29. Jradi, M.; Riffat, S. Tri-generation systems: Energy policies, prime movers, cooling technologies, configurations and operation strategies. Renew. Sustain. Energy Rev. 2014, 32, 396-415. [CrossRef]

30. Bao, Z.; Zhou, Q.; Yang, Z.; Yang, Q.; Xu, L.; Wu, T. A Multi Time-scale and Multi Energy-type Coordinated Microgrid Scheduling Solution-Part I: Model and Methodology. IEEE Trans. Power Syst. 2015, 30, 2257-2266. [CrossRef] 
31. Li, Z.; Jenkins, N.; Wu, W.; Shahidehpour, M.; Wang, J.; Zhang, B. Combined Heat and Power Dispatch Considering Pipeline Energy Storage of District Heating Network. IEEE Trans. Sustain. Energy 2016, 71, $12-22$. [CrossRef]

32. Li, J.; Fang, J.; Zeng, Q.; Chen, Z. Optimal Operation of the Integrated Electrical and Heating Systems to Accommodate the Intermittent Renewable Sources. Appl. Energy 2016, 167, 244-254. [CrossRef]

33. Bloess, A.; Schill, W.; Zerrahn, A. Power-to-heat for renewable energy integration: A review of technologies, modeling approaches, and flexibility potentials. Appl. Energy 2017, 212, 1611-1626. [CrossRef]

34. Dolara, A.; Grimaccia, F.; Magistrati, G.; Marchegiani, G. Optimization Models for Islanded Micro-Grids: A Comparative Analysis between Linear Programming and Mixed Integer Programming. Energies 2017, 10, 241. [CrossRef]

35. Gu, W.; Wang, Z.; Wu, Z.; Luo, Z.; Tang, Y.; Wang, J. An online optimal dispatch schedule for CCHP microgrids based on model predictive control. IEEE Trans. Smart Grid 2017, 8, 2332-2342. [CrossRef]

(C) 2018 by the authors. Licensee MDPI, Basel, Switzerland. This article is an open access article distributed under the terms and conditions of the Creative Commons Attribution (CC BY) license (http://creativecommons.org/licenses/by/4.0/). 